\title{
Influência do treinamento de força em programas de emagrecimento
}

\author{
Strength training influence on weight loss programs \\ Fuerza influencia capacitación en programas de pérdida de peso \\ Daniel CAPRA ${ }^{1}$ \\ Luis Guilherme TARTARO ${ }^{1}$ \\ Roberto Aparecido MAGALHÃES ${ }^{2}$ \\ Anderson MARTELLI ${ }^{3}$ \\ ${ }^{I}$ Graduado em Educação Física pela Faculdade Mogiana do Estado de São Paulo, FMG - Mogi Guaçu-SP, Brasil \\ ${ }^{2}$ Pós-Graduado em Fisiologia do Exercício - UNIFESP, Universidade Federal de São Paulo, São Paulo - SP, Brasil \\ Docente do Curso de Graduação em Educação Física da Faculdade Mogiana do Estado de São Paulo, FMG-Mogi Guaçu-SP, Brasil \\ ${ }^{3}$ Mestrando Programa de Pós-Graduação em Ciências Biomédicas, UNIARARAS, Araras - SP, Brasil \\ Pós Graduado em Laboratório Clínico - Faculdade de Ciências Médicas - UNICAMP, \\ Universidade Estadual de Campinas, Campinas - SP, Brasil; \\ Docente do Curso de Graduação em Educação Física da Faculdade Mogiana do Estado de São Paulo, FMG-Mogi Guaçu-SP, Brasil
}

\begin{abstract}
Resumo
Introdução. A obesidade vem ganhando proporções pandêmicas e tem se tornado um grande problema de saúde pública mundial, acarretando inúmeras consequências de aspectos fisiológicos, insalubres e econômicos. O treinamento de força é uma prática conhecida, mas negligenciada quando o assunto é emagrecimento havendo uma preferência por outras modalidades de exercícios. Objetivos. A presente revisão faz uma abordagem sobre os benefícios do treinamento de força visando à diminuição do percentual de gordura de seus praticantes pelo aumento do gasto calórico e um emagrecimento saudável. Conclusão. A prática do treinamento de força pode ter uma grande influência na perda de peso e torna-se um aliado na recuperação dos componentes básicos da estrutura corporal favorecendo uma melhora da qualidade de vida dos praticantes e a saúde de modo geral.
\end{abstract}

Descritores: Obesidade; Exercício; Perda de Peso.

\begin{abstract}
Introduction. Obesity is gaining epidemic proportions and has become a major public health problem worldwide, resulting in numerous physiological consequences, unhealthy and economic aspects. Strength training is a practice known but neglected when it comes to weight loss there is a preference for other types of exercises. Goals. This review is a discussion of the benefits of strength training aimed at reducing the percentage of fat of its practitioners by increasing energy expenditure and a healthy weight loss. Conclusion. The practice of strength training can have a major influence on weight loss and becomes an ally in the recovery of the basic components of the body structure favoring an improved quality of life for practitioners and the general health.

Descriptors: Obesity; Exercise; Weight Loss.
\end{abstract}

\section{Resumen}

Introducción. La obesidad está adquiriendo proporciones epidémicas y se ha convertido en un importante problema de salud pública en todo el mundo, dando lugar a numerosas consecuencias fisiológicas, insalubres y aspectos económicos. El entrenamiento de fuerza es una práctica conocida pero descuidado cuando se trata de la pérdida de peso no es una preferência por otros tipos de ejercicios. Objetivos. Esta opinión es la discusión de los beneficios del entrenamiento de fuerza destinadas a reducir el porcentaje de grasa de sus practicantes al aumentar el gasto de energía y una pérdida de peso saludable. Conclusión. La práctica de entrenamiento de fuerza puede tener una gran influencia en la pérdida de peso y se convierte en un aliado en la recuperación de los componentes básicos de la estructura del cuerpo que favorecen una mejor calidad de vida para los profesionales y la salud general.

Descriptores: Obesidad; Ejercicio; Pérdida de Peso. 


\section{INTRODUÇÃO}

Nos dias atuais, significativos avanços tecnológicos que fazem parte da rotina das pessoas como a internet, avançados computadores e suas formas portáteis, bem como os telefones celulares que simplificaram diversas atividades diárias do cotidiano sem realizar grandes esforços agregaram inúmeros benefícios às pessoas, especialmente no quesito praticidade. Porém, essas tecnologias possuem certas desvantagens em relação à saúde, uma vez que se substituíram as atividades simples do dia a dia como a locomoção, contribuindo fortemente para o sedentarismo ${ }^{1}$.

Neste contexto, o exercício físico vem ganhando destaque como poderoso elemento na intervenção não farmacológica para a prevenção e tratamento da obesidade, estando bem descrito na literatura que a prática sistemática de atividades físicas exercem ação positiva na redução do peso corporal e nos elementos de risco relacionados à saúde ${ }^{2}$.

Em meio a uma gama de exercícios encontrados hoje, um método que se destaca em relação à melhora da composição corporal é o treinamento de força (TF), que consiste na realização de exercícios com contrações voluntárias da musculatura esquelética, buscando vencer uma resistência, que pode ser desde equipamentos, pesos livres, implementos ou o próprio peso corporal ${ }^{3}$. Para Fleck e Kraemer, o TF contribui significativamente para a diminuição do percentual de gordura corporal, pois ocorre uma ascensão do metabolismo decorrente do aumento de massa muscular, proporcionando ao organismo uma maior oxidação de calorias devido a uma maior demanda energética, mesmo em situação de repouso.

As atividades físicas de forma geral contribuem de maneira positiva para o balanço energético, podendo auxiliar no controle de peso. Neste sentido, o TF se mostra uma ferramenta de auxílio na redução do sedentarismo e aumento do gasto calórico. Assim, esta revisão visa demonstrar a eficiência do TF dentro de programas de emagrecimento, através de repostas do organismo às variáveis de treinamento que visam maximizar a perda de gordura.

\section{MATERIAL E MÉTODO}

Seguindo a metodologia de Matavelli et al. ${ }^{4}$, foi realizado um levantamento bibliográfico nas bases de dados Scielo, Lilacs, Portal de Periódicos da Coordenação de Aperfeiçoamento de Pessoal de Nível Superior (CAPES) e busca de dados no Google Acadêmico de artigos científicos publicados até 2015 utilizando como descritores isolados ou em combinação: treinamento de força, qualidade de vida, obesidade, perda de peso. Adicionalmente, foi realizada consulta de livros acadêmicos para complementação das informações sobre os benefícios do treinamento de força para a terceira idade.

Para seleção do material, efetuaram-se três etapas. A primeira foi caracterizada pela pesquisa do material que compreendeu entre os meses de fevereiro a agosto de 2015. A segunda compreendeu a leitura dos títulos e resumos dos trabalhos, visando uma maior aproximação e conhecimento, sendo excluídos os que não tivessem relação e relevância com o tema e a terceira, a busca dos textos que se encontravam disponíveis na íntegra, sendo estes, inclusos na revisão.

Dos artigos selecionados e incluídos na pesquisa constituíram ensaios clínicos, artigos originais, revisões e revisões sistemáticas da literatura. Como critérios de inclusão dos artigos, analisaram-se a procedência da revista e indexação, estudos que apresentassem dados referentes ao treinamento de força e seus benefícios no emagrecimento publicados entre os anos de 2002 até o mais atual 2015. Como critério de exclusão utilizou-se referência incompleta e informações presentemente desacreditadas, já que essa pesquisa visa revisar conhecimentos atualizados sobre o tema.

\section{○ Aspectos fisiopatológicos da Obesidade e formas de controle}

Atualmente, a obesidade é considerada um dos maiores transtornos de saúde pública em âmbito mundial, sendo caracterizada como uma patologia que favorece o desenvolvimento de inúmeras outras doenças sistêmicas, e com base nestes dados preocupantes, fica claro que medidas de prevenção precisam ser implantadas 5 . A obesidade é definida como um distúrbio nutricional e metabólico onde ocorre um aumento do tecido adiposo ocasionando aumento de peso corporal. Em meio às diversas causas da obesidade, o alto consumo alimentar e o baixo nível de atividade física são uns dos fatores preponderantes para seu desenvolvimento ${ }^{7,8}$, além de fatores genéticos e comportamentais ${ }^{9}$. Cruz et al. ${ }^{10}$ retratam em seu estudo uma significativa associação entre obesidade e o sentimento de insegurança, demonstrando que o fator psicológico pode influenciar na manutenção ou aumento do excesso de peso. Melca e Fortes ${ }^{11}$ descrevem que os transtornos mentais colaboram para o desenvolvimento da obesidade, que por sua vez, aumenta a ocorrência de transtornos mentais como depressão, ansiedade e transtornos alimentares que influenciam diretamente a autoimagem e a insatisfação corporal.

Corroborando com os fatores acima, Libório ${ }^{12}$ cita os elementos socioculturais (estado civil, nível educacional e socioeconômico), comportamentais (consumo de álcool, nutrição, estado psicológico, 
atividade física), alimentação rica em gordura e sedentarismo, adotado por muitos, como determinantes para a obesidade. Juntamente a esses fatores, os avanços da sociedade moderna como a informática, agregaram inúmeros benefícios às pessoas, especialmente no quesito praticidade. Porém essas tecnologias possuem certas desvantagens em relação à saúde, uma vez que, vem ocorrendo à substituição das atividades físicas mais primitivas como a locomoção pelo comodismo, contribuindo fortemente para o sedentarismo ${ }^{1}$.

Segundo a classificação da obesidade, Salve ${ }^{13}$ retrata que esta pode ser classificada em exógena, causada pelo alto consumo de calorias, sendo responsável pela maioria dos casos com 95\%, ou endógena, causada por distúrbios metabólicos e hormonais. No Brasil, de acordo com dados publicados pelo Instituto Brasileiro de Geografia e Estatística (IBGE), a ocorrência de obesidade é de $12,4 \%$ em homens e 16,9\% em mulheres. Já o sobrepeso, atinge metade dos adultos em todas as regiões do país ${ }^{7}$.

Situações parecidas são encontradas entre as crianças brasileiras. Aquelas que se encontram na faixa etária de 5 a 9 anos, estudos demonstram que um terço se encontra acima do peso apropriado segundo a Organização Mundial de Saúde (OMS) ${ }^{14}$. Quanto aos jovens de 10 a 19 anos, os índices chegam a $21,7 \%{ }^{15}$. Em uma pesquisa realizada por Bozza et al. ${ }^{16}$, foi observado que indivíduos jovens menos ativos possuem maiores chances de serem obesos ou de ganharem gordura abdominal, reforçando a necessidade neste grupo da prática de atividades físicas. Uma questão interessante retratada por Lima ${ }^{17}$ é a opção de se morar em apartamentos por causa da violência urbana, onde o espaço é reduzido e as pessoas fazem menos caminhadas e as crianças simplesmente não saem do local para brincar, correr, ou fazer alguma atividade física; o resultado é um gasto calórico menor com efeitos negativos no futuro.

Gentil ${ }^{18}$ expõe que um indivíduo é considerado obeso quando a quantidade de tecido adiposo eleva-se numa proporção que prejudica sua saúde física, resultando em inúmeros problemas no cotidiano, inclusive psicológicos, reduzindo a autoestima, apresentando problemas para o convívio social, familiar e dentro de relacionamentos. O parâmetro definido pela OMS ${ }^{14}$, apud Gentil ${ }^{18}$, estabelece que para definir se um indivíduo se encontra com sobrepeso ou não é utilizado o Índice de Massa Corporal (IMC) Tabela 1, reforçando que este índice é um método bastante prático e rápido que relaciona peso e altura e apresenta uma boa conexão com a quantidade de gordura corporal, mas quando realizado em atletas ou indivíduos que possuem muita massa muscular, apresenta o valor falsamente elevado.
Tabela 1. Classificação, Grau de Obesidade e Risco de comorbidade recomendada pela OMS

\begin{tabular}{|l|c|c|c|}
\hline \multicolumn{1}{|c|}{ IMC $\left(\mathrm{kg} \cdot \mathrm{m}^{2}\right)$} & Classificaçāo & $\begin{array}{c}\text { Grau de } \\
\text { Obesidade }\end{array}$ & $\begin{array}{c}\text { Risco de } \\
\text { Co-morbidade }\end{array}$ \\
\hline Abaixo de 18,5 & Peso Baixo & 0 & Baixo \\
\hline $18,5-24,9$ & Peso Normal & 0 & Médio \\
\hline $25-29,9$ & Sobrepeso & I & Aumentado \\
\hline $30-39,9$ & Obeso & II & Moderado-Alto \\
\hline Acima de 40 & Obeso Grave & III & Altissimo \\
\hline
\end{tabular}

Segundo o Ministério da Saúde ${ }^{19}, 67,3 \%$ das mortes no Brasil em 2007 foram causadas por doenças crônicas não transmissíveis (DCNT), sendo as doenças cardiovasculares responsáveis por $(29,4 \%)$, seguido pelo câncer $(15,1 \%)$, estima-se que em $200863 \%$ das mortes em todo o mundo foram causadas por DCNT, em $30 \%$ das mortes a idade foi inferior a 60 anos. Alguns fatores como obesidade, tabagismo e sedentarismo aumentam as chances de uma pessoa desenvolver tais doenças, como o diabetes. Meneguzzo et al. $^{20}$ relata o ônus gerado para os serviços de saúde, afirmando que as DCNTs exigem tratamentos constantes, com altos custos econômicos e sociais, além de influenciar na execução de atividades importantes, como o absteísmo ao trabalho.

Por outro lado, muitos conceitos estão sendo modificados. Cintra et al. ${ }^{21}$ referem a procura pelo "corpo perfeito" que deixou de ter caráter simplesmente estético, possuindo uma vertente que visa saúde, onde, manter o corpo saudável, tende a diminuir os riscos e incidências de doenças relacionadas com a obesidade. Neste contexto, o exercício físico vem ganhando destaque como poderoso elemento na intervenção não farmacológica para a prevenção e tratamento da obesidade, sendo comprovado que a prática sistemática do exercício exerce ação positiva na redução do peso corporal $^{2,22} \mathrm{e}$ controle das DCNTs, como doenças respiratórias, cardiovasculares, câncer e diabetes ${ }^{23}$.

Arruda et al. $^{24}$ retratam que a combinação de exercício físico e dieta equilibrada proporciona índices negativos na aferição do balanço energético. Desta forma, se o gasto calórico for superior ao consumo energético, o resultado dessa equação será o emagrecimento. Cintra et al. $^{21}$ complementa que é fundamental para a redução do peso corporal o gasto energético resultante da prática do exercício físico, independentemente se o mesmo for realizado em alta ou baixa intensidade.

É interessante demonstrar que reduzir os tecidos adiposos é ter uma atitude de mudanças e ritmo de vida, para não se aumentar a gordura corporal. Entre as alternativas para este controle, temos a prática regular de exercícios físicos e as dietas restritivas, que deveriam ser implantadas na vida das pessoas como ação preventiva ${ }^{18}$. Neste sentido Xavier $^{6}$ retrata que a 
prevenção é o melhor tratamento para obesidade e deve ser preconizado desde a amamentação até a introdução dos primeiros alimentos.

\section{○ Influência do treinamento de força nos programas de emagrecimento}

Historicamente, o TF era realizado por soldados, atletas e pessoas comuns. A literatura nos mostra que em 3600 a. C., na Dinastia Chou na China, os soldados eram submetidos a testes de levantamento de peso para fazer parte do exército. Na Mesopotâmia, encontram-se indícios de proezas e feitos atléticos realizados por homens fortes de épocas antecedentes. $\mathrm{Na}$ Grécia antiga e Índia, o levantamento de pedras era uma prática adotada para promoção da saúde. No entanto, conforme as lendas, Milo de Krotona, seis vezes campeão olímpico de luta, foi o pai do $\mathrm{TF}^{25}$.

Santarém ${ }^{26}$ ressalta que a musculação pode ser um termo utilizado para se referir ao $\mathrm{TF}$ ou treinamento contra resistências graduáveis, reportando seu efeito mais claro, que é o aumento do volume dos músculos estriados esqueléticos e sua capacidade de contração. De acordo com Grahl et al., ${ }^{27}$ é notável que o TF está em constante crescimento, adquirindo confiança e credibilidade em meio a população e entidades importantes, como o American College of Sports Medicine (ACSM). Isto se refere ao seu valor na promoção da saúde, sendo indicado de maneira ampla, desde crianças a idosos, portadores ou não de doenças associadas.

Santarém ${ }^{26}$ relata que o TF vem sendo cada vez mais indicado por estudiosos, pois, além de aumentar a massa muscular, contribui com a aptidão física, melhora a atividade metabólica, flexibilidade, coordenação, composição corporal, proporciona adaptações cardiovasculares importantes para esforços curtos, repetitivos e relativamente intensos, além de auxiliar na prevenção de queda de pessoas idosas. Para Prestes et al. ${ }^{28}$ a prática de $\mathrm{TF}$ diminui a pressão arterial, diminuindo assim os riscos de doenças cardiovasculares, reduz a taxa de colesterol total e glicemia e aumenta a sensibilidade à insulina. Moura e Lima $^{29}$ destacam que o TF com alta intensidade estimula a calcificação e o fortalecimento ósseo, sendo de vital importância para o combate as doenças musculoesqueléticas.

Fahey $^{25}$ demonstra que os benefícios emocionais e sociais gerados pelo TF são significativos. Aspectos como corpo atlético e saudável são extremamente importantes para se melhorar a autoestima. O TF, quando bem orientado, é capaz de moldar o corpo em um curto espaço de tempo e melhorar a sociabilidade, uma vez que ao praticar exercícios na academia, as pessoas se comunicam umas com as outras e criam vínculos, elevando seu quadro social ${ }^{25}$. Os benefícios da prática de exercícios físicos são indiscutíveis, porém a prescrição de TF objetivando o emagrecimento ainda tem sido negligenciada. No entanto, estudos mostram que o TF tem sido um instrumento eficaz na redução de massa gorda ${ }^{30}$

Para Bompa ${ }^{31}$ a importância do TF tem ligação direta com as adaptações de hipertrofia muscular, causando o aumento das miofibrilas e da densidade capilar por fibra muscular, elevando desta forma, a quantidade de proteína e do número total de fibras musculares. Segundo Montenegro ${ }^{30}$ o TF produz um gasto energético durante a sessão, aumento da massa muscular e da taxa metabólica basal (TMB), melhorando a autonomia dos obesos e colaborando assim com os programas de emagrecimento. Esses dados corroboram com os estudos de Fleck e Kraemer $^{3}$ e Grahl et al. ${ }^{27}$, descrevendo que a elevação da TMB é acarretada por aumentos na massa corporal magra, diminuição da porcentagem de gordura corporal e após sessão de TF o metabolismo permanece elevado por várias horas, ampliando a oxidação de lipídeos.

De acordo com Cornelian et al. ${ }^{32}$ para se aumentar a massa muscular é necessário a prática sistemática de exercícios resistidos e exercícios de alta intensidade, já que a falta deste promove um processo inverso ao de hipertrofia muscular. A seleção do método deve incluir exercícios árduos e de alto impacto fisiológico para que as fibras musculares sejam recrutadas em grande quantidade favorecendo um aumento da massa magra. Arruda et al. ${ }^{24}$ reforçam que o TF é significativo para a elevação do gasto energético dos indivíduos em repouso, o que favorece a manutenção e até ampliação dos índices de massa magra.

Um mecanismo fisiológico vem sendo considerado e estudado nas últimas décadas, conhecido como Excess post exercise oxygen consumption (EPOC), e possui uma grande contribuição para o emagrecimento saudável através do treinamento de alta intensidade ${ }^{5}$. Essa resposta fisiológica está sendo muito investigada em indivíduos após os treinamentos, uma vez que os níveis de oxigênio se mantêm aumentado ao fím dos exercícios. $\mathrm{O}$ treinamento resistido entra como uma vertente dos exercícios de alta intensidade, nos quais durante a atividade, os estoques de glicogênio são depletados fazendo com que o organismo tenha que repô-los, e para que isso ocorra, à oxidação de gordura é aumentada buscando preservar a glicose ${ }^{5}$.

Segundo Lima-Silva et al. ${ }^{33}$, é respaldado pela ciência que exercícios de curta duração e baixa intensidade não são satisfatórios para produzir um EPOC duradouro e de elevada magnitude. Ambos, intensidade e duração de esforço, são os fatores primordiais que definem a duração e magnitude do EPOC. A duração e magnitude do EPOC se ampliam 
exponencialmente em atribuição da intensidade, enquanto ampliam de forma linear em atribuição da duração do esforço. Esforços de grande intensidade (supra máximos) e de curta duração se mostram eficientes para produzir EPOC com valores elevados $^{33}$.

$\mathrm{Na}$ visão prática, o exercício físico pode colaborar em programas de emagrecimento, não apenas por elevar o gasto energético durante o exercício, mas também por contribuir com o aumento do gasto energético durante a fase de recuperação ${ }^{33}$. Reforçando a parte prática, Pinto et al. ${ }^{34}$ expõe que exercícios que envolvem grandes grupos musculares, elevada intensidade, alto volume de treinamento e curtos intervalos de recuperação geram um gasto energético aumentado de maneira aguda no exercício.

Escurra et al. $^{35}$ realizaram uma pesquisa em uma academia de Campo Grande (MS) com 15 mulheres sedentárias e iniciantes na modalidade, com faixa etária entre 18 a 30 anos e por um período de 12 semanas. O trabalho foi realizado três vezes por semana durante 1 hora por dia. Ao final, foi verificado que o TF foi capaz de gerar um gasto energético necessário para promover a oxidação da adiposidade subcutânea e manutenção da massa corporal magra.

Um estudo, utilizando também o público feminino, conduzido por Teixeira e Rocha ${ }^{36}$ analisou um grupo de 20 mulheres com idade entre 50 e 60 anos, por um período de 14 semanas em um programa de 36 sessões. Os resultados demonstraram que o TF de maneira periodizada promoveu adaptações positivas quanto à diminuição do percentual de gordura e índice de massa corporal em relação cintura/quadril.

Na pesquisa de Porto et al. ${ }^{37}$, foi avaliado como a utilização do TF pode interferir na composição corporal e na força muscular em um grupo de 9 crianças do sexo masculino, obesas, com idade entre 9 e 10 anos, durante 10 semanas com intensidade entre 45 a $65 \%$ de 10 repetições máximas (RM), frequência de 3 vezes por semana com duração de 50 minutos. Os autores observaram alterações positivas na composição corporal com a diminuição da massa gorda associada ao aumento da força muscular.

Nunes e Sousa ${ }^{38}$ realizaram uma pesquisa para averiguar o efeito do TF durante 12 sessões com intensidade de $60 \%$ de $1 \mathrm{RM}$ em uma mulher adulta e sedentária. Os resultados demonstraram que o TF aplicado diminuiu a porcentagem de gordura corporal e o aumento da massa magra causando um impacto sobre a composição corporal do sujeito da pesquisa como demonstrado na figura 1.

A prática regular de exercícios físicos favorece inúmeros benefícios que se manifestam sob todos os aspectos do organismo. $\mathrm{Na}$ saúde, está bem estabelecido a perda de peso e porcentagem de gordura corporal, melhora do diabetes, diminuição do colesterol total e o favorecimento do controle da pressão arterial $^{4}$.

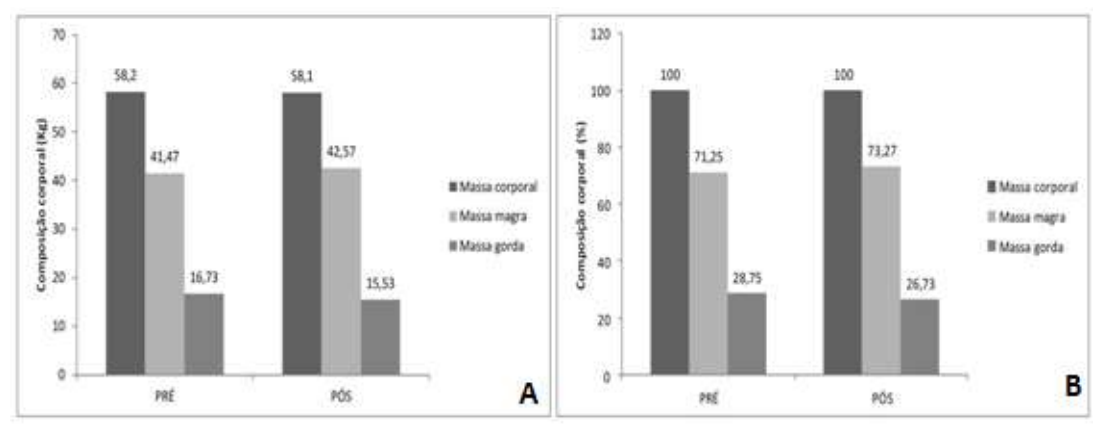

Figura 1. Em A, Composição corporal expressa em quilogramas; B, Composição corporal expressa em porcentagem. Extraído e modificado de Nunes e Sousa ${ }^{38}$

Apesar de inúmeros estudos e incentivos à prática do $\mathrm{TF}$, ainda estamos longe de atingir em totalidade esse público. Muitas dessas pessoas encontram limitações para este tipo de atividade sejam eles os fatores econômicos, sociais, políticas governamentais e a falta de maior informação por parte da população ${ }^{39}$.

Os estudos, as pesquisas e os incentivos à prática do TF direcionado a perda de peso devem ser continuados, com o intuito de demonstrar a população o quanto são importantes esses métodos de exercício e como as pessoas podem melhorar suas capacidades através de seus benefícios respeitando sempre o critério da individualidade.

\section{DISCUSSÃO E CONCLUSÃO}

Através dessa revisão pode-se observar que a obesidade nos dias atuais é considerada um problema de saúde pública e em constante evolução, com causas bem definidas, sendo a mudança no estilo de vida das pessoas um fator atenuante. Este quadro favorece o desenvolvimento de várias outras patologias como as DCNT, afetando diretamente a saúde do indivíduo com redução de sua qualidade de vida e o ônus que o governo possui para com esses indivíduos, uma vez que essas doenças necessitam de tratamentos constantes, e geram altos custos econômicos.

Foi verificado que o treinamento de força juntamente com uma dieta alimentar balanceada pode produzir efeitos positivos na perda de peso, impactando diretamente no balanço energético do indivíduo. Hoje, este tipo de exercício vem ganhando maior credibilidade na população de modo geral pelas diversas pesquisas que vem sendo desenvolvida nos últimos anos.

Apesar das limitações deste estudo, conclui-se que o TF pode contribuir para a melhoria da composição corporal, mas como todo exercício, este deve ser prescrito por um profissional capacitado, uma vez que o TF possui inúmeras variáveis, e cabe ao profissional de educação física adaptar à individualidade de cada cliente. 
Futuros estudos devem ser realizados visando o aperfeiçoamento de técnicas, além de buscar expor novas contribuições por se tratar de um assunto amplo e de grande importância mundial.

\section{REFERENCIAS}

1. Mendes CML, Cunha RCL. As novas tecnologias e suas influências na prática de atividade física e no sedentarismo. Revista Interface: Saúde, Humanas e Tecnologia. 2013;1(3):s/p.

2. Dias IBF, Montenegro RA, Monteiro WD. Exercícios físicos como estratégia de prevenção e tratamento da obesidade: aspectos fisiológicos e metodológicos. Rev HUPE. 2014; 13(1):70-9.

3. Fleck SJ, Kraemer WJ. Fundamentos do treinamento de força muscular. 3.ed. Porto Alegre: Artmed; 2006.

4. Maravelli IS, Del Judice EL, Matavelli R, Martelli A. Hipertensão Arterial Sistêmica e a Prática Regular de Exercícios Físicos como Forma de Controle: Revisão de Literatura. RBCS. 2014;18(4):359-66.

5. Silva Filho JN. Treinamento de força e seus benefícios voltados para um emagrecimento saudável. RBPFEX. 2013; 7(40):329-38.

6. Xavier BMLS. Fatores relacionados à obesidade infantil [trabalho de conclusão de curso]. Valparaiso: Faculdade de Ciências da Educação Sena Aires; 2014.

7. Sá JM, Melo MR, Speretta GFF, Menani JV, Colombari E, Colombari DSA. Bloqueio de receptores AT1 centrais reduz a pressão arterial em animais alimentados com dieta hiperlipídica. Rev Ciênc Farm Básica Apl. 2015; 36(1):s/p.

8. Hernades F, Valentini MP. Obesidade: Causas e consequências em crianças e adolescentes. Conexões. 2010;8(3):47-63.

9. Buff CG, Ramos E, Souza FIS, Sarni ROS. Frequência de síndrome metabólica em crianças e adolescentes com sobrepeso e obesidade. Rev Paul Pediat. 2007; 25(3): 221-6.

10. Cruz SH, Zanon RB, Bosa CA. Relação entre Apego e Obesidade. PSICO. 2015;46(1):6-15.

11. Melca IA, Fortes S. Obesidade e transtornos mentais: construindo um cuidado efetivo. Rev HUPE. 2014; 13(1):18-25.

12. Libório MFRA. Ambientes obesogénicos: Casa, área de residência e escola [dissertação de mestrado]. Vila Real: Universidade de Trás-osMontes e Alto Douro; 2010.

13. Salve MGC. Obesidade e peso corporal: riscos e consequências. Movimento \& Percepção. 2006; 6(8):29-48.

14. World Health Organization. WHO report on the global tobacco epidemic, 2011: warning about the dangers of tobacco. Geneva: WHO, $2011 \mathrm{~b}$. Disponível em: <http://www.who.int/tobacco/ global_report/2011/en/> [Acesso em 24 Out. 2015].

15. CONFEF. O futuro da Humanidade. Revista EF. 2012; 10(43). Disponível em: <http://www.confef.org.br /extra/revistaef/ show. asp?id=3995> [Acesso em 10 Out. 2015].

16. Bozza R, Campos W, Bacil EDA.; Filho VCB, Hardt MJ, Silva PM. Fatores sociodemográficos e comportamentais associados à adiposidade corporal em adolescentes. Rev Paul Pediat. 2014; 32(3):241-6.

17. Lima RM. Obesidade, o mal do século. Revista Perspectiva Online. 2007; 1(2):86-99.

18. Gentil P. Emagrecimento - Quebrando Mitos e Paradigmas. Rio de Janeiro, Sprint, 2011.

19. BRASIL. Ministério da Saúde. Secretaria de Vigilância em Saúde. Departamento de Análise de Situação de Saúde. Plano de ações estratégicas para o enfrentamento das doenças crônicas não transmissíveis (DCNT) no Brasil 2011-2022 / Ministério da Saúde. Secretaria de Vigilância em Saúde. Departamento de Análise de Situação de Saúde. - Brasília: Ministério da Saúde, 2011.

20. Meneguzzo C, Ricalde SR, Santos JS, Mendes KG. Prevalência de sobrepeso e obesidade em crianças atendidas em uma Unidade Básica de Saúde no Município de Antônio Prado (RS). Cad. Saúde Colet. 2010,18 (2):275-81

21. Cintra DE, Ropolle ER, Pauli JR. Obesidade e Diabetes: Fisiopatologia e Sinalização Celular. São Paulo: Sarvier, 2011.

22. Pontes ALC, Sousa IA, Navarro AC. O tratamento da obesidade através da combinação dos exercícios físicos e terapia nutricional visando o emagrecimento. RBONE. 2009; 3(14):124-35.

23. Warschauer M, Carvalho YM, Martins CL, Freitas FF. As escolhas das práticas corporais e dos profissionais que as conduzem nas unidades básicas de saúde do distrito Butantã/SP. In: $15^{\circ}$ Congresso Brasileiro de Ciências do Esporte, II Congresso Internacional de Ciências do Esporte.Recife, 2007.

24. Arruda DP, Assumpção CO, Urtado CB, Dorta LNO, Rosa MRR, Zabaglia R, Souza TMF. Relação entre treinamento de força e redução do peso corporal. RBPFEX. 2010;4(24):605-9.

25. Fahey TD. Bases do treinamento de força para homens e mulheres. 8.ed. Nova York: AMGH; 2013.

26. Santarém JM. Musculação em todas as idades: comece a praticar antes que seu médico recomende. Barueri: Manole; 2012.

27. Grahl G, Noamann JT, Nunes RH, Flores LJF. 
Efeitos do Treinamento Resistido na redução do percentual de gordura corporal em adultos: Uma revisão de literatura. CEFE. 2013;11(2):69-77.

28. Prestes J, Foschini D, Marchetti P, Charro M. Prescrição e Periodização do Treinamento de Força em Academias. São Paulo: Manole; 2010.

29. Moura ECC, Lima YS. O treinamento de força e seus possíveis benefícios em pacientes com osteoporose. EfDeportes.com. 2010;15(148). Disponível em: http://www.efdeportes.com/ efd148/o-treinamento-de-forca-em-pacientes-comosteoporose.htm.

30. Montenegro LP. Musculação: Aspectos positivos para o emagrecimento. RBPFEX. 2014; 8(43):100-5.

31. Bompa OT. Teoria e Metodologia do Treinamento. 4.ed. São Paulo:Phorte; 2002.

32. Cornelian BR, Moreira J, Oliveira HG. Intensidade do treinamento para ganho de massa magra: Revisão de métodos para orientação prática. Rev UNINGÁ Review. 2014;18(3):37-43.

33. Lima-Silva AE, Pires FO, Bertuzzi R. Excesso de oxigênio consumido pós-esforço: Possíveis mecanismos fisiológicos. Reveducfis. 2010;21(3):563-75.

34. Pinto RS, Lupi R, Brentano MA. Respostas metabólicas ao treinamento de força: uma ênfase no dispêndio energético. Rev bras cineantropom desempenho hum. 2011;13(2):150-7.

35. Escurra M, Santiago AP, Ovando RGM. A musculação como um dos fatores determinantes para diminuição do percentual de gordura corporal em praticantes do sexo feminino. Fiep Bulletin. 2014;84(Spec Iss). Disponível em: http:// www.fiepbulletin.net)

36. Teixeira AV, Rocha GM. Efeito de um protocolo de treinamento de força periodizado sobre a composição corporal de mulheres com idades entre 50 e 60 anos. RBPFEX. 2014;8(44).

37. Porto M, Nagamine KK, Brandão AC, Florim GS, Pinhel MA, Souza EO, Souza DRS. Programa de treinamento resistido sobre a composição corporal e na força muscular de crianças com obesidade. $\mathrm{R}$ bras Ci e Mov. 2013;21(4):21-9.

38. Nunes FB, Sousa EN. Efeito de 12 sessões de treinamento resistido na composição corporal: um estudo de caso. RBPFEX. 2014;8(49):674-9.

39. Lopes CDC, Magalhães RA, Hunger MS, Martelli A. Treinamento de força e terceira idade: componentes básicos para autonomia. Arch Health Invest. 2015;4(1):37-44.

\section{CONFLITO DE INTERESSES}

Os autores declaram não haver conflitos de interesse.

\section{AUTOR PARA CORRESPONDÊNCIA}

Anderson Martelli

martellibio@hotmail.com
Submetido em 02/12/2015 Aceito em 11/12/2015 